\title{
Téoros
}

Revue de recherche en tourisme

\section{Tourisme et femmes}

\section{Véronique Antomarchi et Suzanne De La Barre}

Volume 29, numéro 2, 2010

\section{Tourisme et femmes}

URI : https://id.erudit.org/iderudit/1024874ar

DOI : https://doi.org/10.7202/1024874ar

Aller au sommaire du numéro

\section{Éditeur(s)}

Université du Québec à Montréal

\section{ISSN}

0712-8657 (imprimé)

1923-2705 (numérique)

Découvrir la revue

\section{Citer ce document}

Antomarchi, V. \& De La Barre, S. (2010). Tourisme et femmes. Téoros, 29(2),

87-92. https://doi.org/10.7202/1024874ar d'utilisation que vous pouvez consulter en ligne.

https://apropos.erudit.org/fr/usagers/politique-dutilisation/ 
PRÉSENTATION

\title{
Tourisme et femmes
}

\author{
Véronique ANTOMARCHI \\ Docteure en histoire \\ Chercheure associée Cerlom-Inalco, Paris \\ veranto@club-internet.fr \\ Suzanne DE LA BARRE, Ph.D. \\ Chercheure associée, postdoctorat \\ Kulturgeografiska institutionen / Département de géographie sociale et économique \\ Université Umeå \\ suzanne.delabarre@geography.umu.se
}

Aujourd'hui, l'importance du genre dans les études portant sur le tourisme n'est plus un sujet nécessitant un débat. En effet, depuis les années 1990, une littérature conséquente montre l'importance du genre dans ce domaine. La relation entre genre et tourisme peut être pensée de différentes façons. Annette Pritchard et Nigel Morgan (2000) reprennent les propositions antérieures de Rao (1995) en affirmant que le lien le plus notable entre le genre et le tourisme provient du fait que le tourisme apparait comme le produit d'une société "genrée». Le développement du tourisme reflète bien les relations entre les sexes ainsi que leurs variations dans le temps et dans l'espace. Moins d'une décennie plus tard, Pritchard et al. (2007: 1), tout en montrant le rôle important joué par le féminisme sur la question du genre et du tourisme, se demandent désormais si, à l'ère du postmodernisme, féminisme et analyse de genre ne seraient pas dépassés, voire obsolètes :

It seems as though before we have really begun to unpick the complexities and implications of gender (and certainly before many research fields - including tourism - have engaged with the nuances of masculinities), Western societies have already become bored by issues of femininities, masculinities and genders. Yet, quite clearly, whatever our social or geographic location, whilst our experiences of (dis) empowerment and (in)equalities may vary, none of us live in equal societies and it is a worrying reality that not one country has yet managed to eliminate the gender gap.

Nous vous proposons un sommaire critique, quoique partiel, car centré sur les pays anglophones et essentiellement sur la France, de la recherche qui a été faite jusqu'à présent sur cette thématique.

Dans le monde anglophone (une catégorisation maladroite qui comprend le Canada, les États-Unis, le Royaume-Uni, l'Australie et la Nouvelle-Zélande), Vivian Kinnaird et Derek Hall ont écrit Tourism: A Gender Analysis (1994). Ce livre est l'un des premiers ouvrages publiés qui traite du genre et du tourisme. Les auteurs ont accordé une attention toute particulière au tourisme comme vecteur du développement économique. En 1995, Margaret Byrne Swain, rédactrice invitée pour le numéro spécial d'Annals of Tourism Research, portant sur le genre et le tourisme, montre que les différents articles mettent l'accent à la fois sur les points communs, mais aussi sur les caractéristiques spécifiques des expériences touristiques de genre et sur la façon dont les questions ont été examinées et expliquées au fil du temps. Margaret Byrne Swain et Janet Henshall Momsen avec Gender/Tourism/Fun? (2002) explorent le genre et le tourisme dans une double perspective : celle du visiteur (le genre et la consommation du tourisme) et celle des hôtes (le genre et les producteurs du tourisme). Les auteurs pensent que la différence entre les sexes fournit un aperçu unique sur les inégalités sociales et apporte une contribution très pertinente au développement théorique. Byrne Swain et Henshall Momsen accordent une attention toute particulière aux avantages de la recherche sur le genre appliquée au tourisme. Elles insistent sur le fait que "as women constitute a growing segment of recreational travel, research on women tourists has a strong applied potential for improved tourism marketing, policy and planning» (Byrne Swain et Henshall Momsen, 2002 : vii).

Le travail d'Annette Pritchard et de divers co-auteurs a apporté une contribution exceptionnelle aux études sur le tourisme. Un article paru dans les Annals of Tourism Research par Annette Pritchard et Nigel Morgan (2000), intitulé «Privileging the Male Gaze: Gendered Tourism Landscape», montre de manière pionnière l'impact de la géographie culturelle et des perspectives féministes sur les recherches portant sur le tourisme et les loisirs. L'utilisation des conceptualisations émergentes de l'espace et du lieu ("place» en anglais) apparaît comme une construction culturelle complexe. Pritchard et Morgan ont analysé la langue patriarcale du marketing en tourisme et ont décrit les caractéristiques de paysages féminisés, utilisés dans la promotion du tourisme. Gender and Sexuality in Tourism Research (Pritchard, 2005) et Tourism and Gender: Embodiment, Sensuality and Experience (Pritchard et al., 2007) offrent un 
aperçu plus exhaustif et critique des études et des développements concernant genre et tourisme. En outre, Pritchard et al. (2007) introduisent une terminologie spécifique axée sur "gender-focused» et «feminist-oriented » qui s'avère très utile à la recherche, en accordant une attention au sexe (les femmes et les hommes) tout en faisant une analyse critique des approches hégémoniques qui (re)produisent et (re)construisent la masculinité et les dispositions patriarcales dans la manière de voir et de comprendre le monde.

Un dernier livre doit être ajouté à cette liste : Gendered Journeys, Mobile Emotions de Gale Letherby et Gillian Reynolds (2009). Cette publication vise à examiner les liens entre l'émotion, le sexe et le voyage. Cet ouvrage est particulièrement pertinent parce qu'il fait le lien entre le tourisme, le genre et l'émergence des " mobility studies». Les études sur la mobilité visent à mettre en avant les interférences entre voyage, transport, tourisme et migration. Elles ouvrent la voie à un examen des déplacements volontaires, motivés par le loisir et le plaisir, mais elles s'intéressent aussi aux déplacements répondant à des motivations plus complexes et néfastes.

Dans le monde francophone, le nombre de publications sur les études de genre ne cesse de croître dans la plupart des disciplines des sciences sociales, comme le montre la parution toute récente de deux ouvrages collectifs dressant le bilan des recherches sur le genre et sur la division des sexes (ChabaudRychter et al., 2010; Riot-Sarcey, 2010). Pourtant, la visibilité de la recherche scientifique concernant le champ qui fait l'objet de ce dossier "tourisme et femmes» apparait faiblement. Ce décalage entre les deux mondes serait peut-être le reflet d'une différence de maturité à penser l'évolution des champs disciplinaires, dans les études sur le genre et sur le tourisme (Pour une discussion sur l'état des études en tourisme avec quelques références aux écarts attribuables à la géographie et à la langue, nous vous référons au numéro spécial de Téoros sur ce sujet lancé au printemps 2008.).

Tout d'abord, la question spatiale du genre a été posée récemment, à l'horizon des années 2000, par plusieurs géographes français. Jean-Luc Piveteau (1996) analyse « [1] a sexuation, principe de synergie territoriale». Elle s'apparente à une géographie "postmoderniste» qui renouvelle à la fois ses objets et ses approches, mais qui connaît un retard assumé en France peut-être parce que «les géographes français sont dans leur majorité des hommes blancs hétérosexuels, a priori peu sensibles aux questions posées par le postmodernisme» (Staszak et Collignon, $2004: 40$ ). Ce même constat interpelle les travaux de Claire Hancock (2004) et de Marianne Blidon (2008) qui s'interrogent avec pertinence sur l'importance de l'appartenance sexuelle dans l'approche scientifique des objets de recherche et des enquêtes de terrain.

C'est d'ailleurs par le biais de la sexualité et du rapport au corps que la réflexion sur le genre en sciences sociales permet d'aborder souvent de manière indirecte et détournée la question du tourisme. Nous pouvons citer le travail de Corinne CauvinVerner (2009) sur les relations sexuelles de touristes occidentales avec des guides dans le Sahara marocain, qui renversent dans une certaine mesure les positions de domination instituées par la situation touristique : «[L]a séduction des étrangères est capitalisée et elle est l'objet d'une compétition, notamment parce qu'elle permet de s'enrichir, de renégocier sa marginalité et même d'émigrer» (Cauvin-Verner, 2009 : 142). De même, Jaurand et De Luze (2004), en étudiant les questions de la nudité et du naturisme, s'intéressent à des espaces touristiques spécifiques et notamment aux plages. On voit bien à travers ces exemples comment «l'érotisation des corps construit aussi l'espace» (Volvey et Stock, $2003:$ :837-838).

Malgré quelques articles ou revues comme l'ouvrage Le genre des territoires, dirigé par l'historienne Christine Bard (2004), ou le numéro spécial de Géographie et Cultures sur le genre, piloté par Francine Barthe et Claire Hancock (2006), force est de constater que les réflexions sur les géographies des genres restent encore marginales en France, par exemple. Saisir la division sexuée de l'espace permettrait pourtant entre autres de renouveler la lecture que l'on peut se faire de la société (Labourie-Racapé, 2004) et de comprendre l'organisation d'une reproduction des inégalités (Blidon et Roux, 2009). Un colloque vient d'être organisé en septembre 2010 en ce sens à l'Université de Bordeaux, «Masculin/Féminin, question pour la géographie».

Alors que nous venons de montrer que plusieurs articles ou revues ont été publiés sur le genre, la question du tourisme et des femmes fait l'objet de plus rares publications encore dans le monde francophone. Elle apparaît même d'une certaine façon comme le point aveugle de la recherche sur le tourisme en France. Ainsi, dans l'éditorial du numéro spécial de la revue Clio consacré aux «Voyageuses», Rebecca Rogers et Françoise Thébaud (2008) constatent que si les femmes ont toujours voyagé ou, pour être plus précises, si elles se sont toujours déplacées, en revanche, «l'histoire des voyages au féminin reste encore dans l'enfance dans les études francophones» (Rogers et Thébaud, $2008: 6$ ). Nous pouvons renchérir en indiquant que l'histoire du tourisme et des femmes serait en état de gestation, surtout chez les chercheurs francophones.

Nous nous heurtons donc à un double retard français concernant à la fois les études sur le genre et sur le tourisme. Dans le monde scientifique, le tourisme reste le monopole quasi exclusif des économistes et des géographes (Cazes, 1992; l'équipe MIT, 2002) jusqu'au début des années 2000. Les anthropologues et les sociologues s'y intéressent tardivement, surtout à partir de 2005, alors que, dans les pays anglophones, les recherches sur le tourisme commencent dès les années 1970 (Cousin et Réau, 2009). Le tourisme a d'ailleurs longtemps été perçu de façon ironique, comme un sujet peu sérieux et moins noble que d'autres thématiques. Le touriste, considéré comme l'idiot du voyage dans un ouvrage pionnier en France, ne suscitait pas ou peu d'intérêt (Urbain, 1991). Pourtant, aujourd'hui, cette activité humaine apparaît de plus en plus comme une clef de lecture indispensable pour comprendre notre monde et «une entrée pertinente pour analyser sous un jour nouveau différents aspects des sociétés contemporaines» (Cousin et Réau 2009).

Plusieurs historiens français se sont penchés sur la question du tourisme et des loisirs à la fin des années 1990 (Boyer, 1999; Corbin, 1995; Rauch, 1996; Bertho-Lavenir, 1999). Ces travaux ont permis une périodisation de l'activité touristique, qui ne fait plus l'objet de discussion. Le tourisme apparaît dans un contexte spatio-temporel bien précis. Comme le souligne Mathis Stock, géographe (2003:20), «l'irruption précoce du 
marché et des méthodes industrielles dans la sphère du tourisme au cours du XIX ${ }^{\mathrm{e}}$ siècle marque l'opposition entre les pratiques aristocratiques héritées de l'otium antique et les pratiques bourgeoises ».

La mise en contexte de la question «femmes et tourisme» dans le monde de la recherche francophone en sciences sociales révèle que nous touchons là à un domaine encore marginal. C'est ce qui en fait aussi tout l'intérêt.

Nous venons d'énumérer partiellement les points de vue francophones et anglophones. Les perspectives féministes et l'analyse des genres permettent de s'écarter des perspectives hégémoniques sur de nombreux thèmes liés au tourisme. De plus, elles mettent en évidence la façon dont le monde est perçu et vécu différemment par tous les «autres» qui ne se reconnaissent pas dans la valeur neutre des hommes. Cette analyse féministe apporte un éclairage nouveau aux études sur le tourisme en tant que discipline ou champ d'étude, peu importe la façon d'entrer dans ce débat (et nous n'y entrerons pas ici). En effet, cette analyse suscite une réflexion susceptible d'apporter une nouvelle approche, à la fois sur les questions de mobilité et d'identité, qui apparaissent comme les deux dimensions centrales de l'évolution des études du tourisme, conceptualisées dans d'autres disciplines plus établies et plus reconnues : géographie, sociologie, histoire, anthropologie, économie. L'analyse féministe et de genre dans le tourisme a donc contribué à démontrer que le tourisme ne se résume pas à un domaine d'études visant seulement à comprendre les affaires du tourisme et la gestion des touristes et de leurs voyages.

Ainsi, l'attention accordée au genre dans les études sur le tourisme contribue également à sa maturité méthodologique. Phillimore et Goodson (2004) soulignent à quel point le genre et l'analyse féministe ont permis aux chercheurs en tourisme qualitatif d'analyser ce que Denzin et Lincoln (1994 et 2000) décrivent comme les préoccupations du «third moment». Le «troisième moment» se situe dans un paradigme postpositiviste influencé par des perspectives qualitatives telles que l'herméneutique (où les accords précédents et les préjugés forment le processus d'interprétation) et les études culturelles (un domaine interdisciplinaire qui combine la théorie critique, le féminisme et le poststructuralisme). Le troisième moment a influencé la façon dans laquelle le tourisme a été capable de considérer le «critical turn» ou le «tournant critique» dans les sciences sociales.

Le tournant critique visait à apporter un changement paradigmatique dans les sciences sociales et a été influencé par la théorie poststructuraliste et critique (Ateljevic et al., 2002). Sans doute, l'attention portée aux préoccupations féministes et de genre, mais aussi les perspectives et les conceptualisations dans le tourisme, ont permis aux études de tourisme d'appréhender le sens critique, d'une manière similaire à ce qui était connu plus généralement dans les sciences sociales. Cette opinion est partagée par Pritchard (2005 : 317) qui affirme que "a deeper insight into the manifestation of gender and sexuality in tourism worlds can contribute to the epistemological enrichment of tourism research». En effet, les contributions féministes permettent aux études sur le tourisme d'explorer divers fondements épistémologiques et d'orienter la façon dont les préoccupations et les questions du tourisme sont conceptualisées (pour une discussion et un excellent résumé sur ce sujet, voir Pritchard et al., 2007, dans la section intitulée "Gender and Tourism Academy », p. 4-6).

Ce numéro de Téoros vise à compléter les études scientifiques du tourisme qui se penchent sur les rapports entre genre et tourisme en développant une recherche sur le tourisme qui soit "gender-focused» et "feminist-oriented» selon les expressions de Pritchard et al. (2007). La présentation synthétique de l'état de la recherche sur ce thème dans les mondes francophone et anglophone a pour vocation d'être un outil pratique.

Néanmoins, même s'il peut paraître novateur dans sa thématique, du moins dans le monde francophone, ce dossier l'est de façon toute relative. En effet, voilà 15 ans, un numéro de Téoros développait exactement ce même thème. Dans sa présentation, Sylvie Gagnon (1997) insistait déjà sur la faible littérature sur le sujet, ce qui l'avait d'ailleurs conduit à retarder le projet de publication pendant sept ans. Les thématiques portaient alors sur les questions de pouvoir ainsi que sur l'emploi féminin dans le secteur du tourisme, dans une perspective féministe nettement plus affichée que ce que nous pouvons lire aujourd'hui. Le présent numéro compte quatre articles se déclinant autour de trois thèmes principaux.

Le premier thème qui se dégage est celui de l'emploi féminin et des rapports de genre dans une région touristique du Mexique. Le second thème porte sur le poids des représentations féminines, d'une part dans les discours associés aux images d'un produit spécifique dédié aux femmes, celui du bien-être, et d'autre part dans les affiches de promotion touristique de la côte normande. Le troisième thème dresse le portrait d'une femme singulière, Ella Maillart, incarnant «un nouveau genre de voyageuse».

Ces quatre textes ne prétendent en aucun cas couvrir ce vaste champ qui apparaît comme un terrain en friche mais, par leurs approches, ils livrent des pistes intéressantes. Ils proviennent d'Espagne, de France, du Québec, ce qui permet de faire entendre une autre voix que celle des études anglophones.

Andrée-Ann Corbin nous présente les résultats d'une enquête de terrain qu'elle a menée dans le cadre de sa mầtrise en anthropologie à l'Université Laval dans la commune de Ciudad Chemuyil au Mexique. Elle montre comment le tourisme transforme le milieu et influe sur les rapports de genre. Exercer une activité professionnelle dans le secteur du tourisme a des conséquences qu'il est important de souligner de manière plus générale. Le secteur touristique est marqué par une très forte féminisation, avec par exemple $70 \%$ de femmes employées dans les agences de voyages et $85 \%$ de femmes travaillant dans les offices de tourisme en France. Pourtant, celles-ci n'occupent pas les postes de direction et continuent de subir des inégalités de traitement. Un rapport récent de l'ONU (2009) met en avant cette situation. Rappelons-nous que la journée mondiale du tourisme, organisée par l'Organisation mondiale du tourisme en 2007, était intitulée «Tourisme, une porte ouverte pour les femmes».

Plusieurs associations de femmes travaillant dans ce secteur se créent dans les mondes francophone et anglophone. Ainsi, l'association Femmes du tourisme, fondée en 2005 en France, a pour objectif de travailler à plus d'égalité dans un monde où la concentration capitalistique est particulièrement forte, ce qui 
limite aussi les postes de pouvoir (entretien Christine Pioli, octobre 2010). De même, Women in Tourism International Alliance Ltd. (WITIA) est un réseau pour les professionnelles du tourisme dans le but notamment de protéger les femmes et les enfants.

Marie-Ange Bugnot, Carmen Cortés et Isabel Turci sont professeures à l'Université de Malaga en Espagne. Elles ont étudié un corpus important de deux magazines de voyage, publiés en France au début des années 2000. Utilisant une approche sémiologique, elles décryptent les discours et les images associés au tourisme de bien-être, analysé ici comme un produit spécifiquement féminin conçu de façon ambivalente pour une femme à la fois passive et dominante car prescriptive de la prestation.

La question de la représentation de la femme est au cœur du texte de trois chercheurs en sciences du sport: Gilles Pécout (Université de Lille 2), Ludovic Birot (Université de Lille 2) et Anaïs Bohuon (Université de Paris XI). L'étude d'une centaine d'affiches de promotion touristique des stations balnéaires normandes entre 1880 et 1960 montre l'omniprésence féminine, à tel point que, selon les auteurs, la femme symboliserait le tourisme balnéaire. Or, la promotion touristique repose sur une mise en désir des territoires, souvent associée à la présence d'un corps féminin. Les stations de montagne mettent en scène à la même époque l'image d'une femme nouvelle, s'adonnant aux sports d'hiver. Ainsi, le catalogue d'une exposition récente d'affiches touristiques de 1860 à 1960 en France insiste sur la présence féminine systématique dans la promotion des stations thermales, balnéaires, de montagne et dans les espaces ruraux (Chevrel, Cornet et Rauch, 2006). Claire Hancock (2004 : 170) évoque le lien particulier de certains géographes à la terre en affirmant que «c'est bien une relation érotisée, la relation d'un sujet masculin à une terre féminisée, qui prend même une figure maternelle, qui se fait jour; pour ces géographes œdipaux, la terre est une femme qu'on habite». Nous pouvons la suivre en interrogeant notre propre relation, homme ou femme, à la terre. Terre, ville, rue, destination, station, plage, mer, montagne sont en français des mots du genre féminin, ce qui dans une certaine mesure accentuerait cette féminisation du territoire. Ces analyses rejoignent les travaux anglophones d'Annette Pritchard et Nigel Morgan (2000:886) qui concluent que «landscapes are shaped by the discourses of patriarchy and (hetero)sexuality and the language of tourism promotion is scripted for a male heterosexual audience».

Comme le montrent Christophe Pécout, Ludovic Birot et Anaïs Bohuon dans leur article, cette représentation de la femme émancipée correspond à une vision masculine fantasmée et s'écarte des réalités sociales de l'époque. La femme fortement soumise aux codes moraux reste une mineure aux plans civil et juridique. Pourtant, les affiches publicitaires commanditées par des compagnies de chemin de fer et des syndicats d'initiative s'adressent plus particulièrement aux mères de famille qui viennent profiter avec leurs enfants des vertus de la mer guérisseuse, puis des bienfaits du soleil (Antomarchi, 2007; Andrieu, 2008; Ory, 2008). Une enquête sociologique menée par Kaufmann (1995) sur les seins nus sur la plage a permis d'analyser les règles du jeu de séduction entre les façons dont les femmes donnent à voir leur corps et dont elles sont regardées par les hommes.

La question des vacances en famille mériterait une exploration plus précise pour construire cette histoire du tourisme au féminin. Nicole Samuel (1997) s'est intéressée de manière pionnière dans ses enquêtes de terrain au vécu des femmes en vacances et notamment à la reproduction de tâches domestiques. En effet, ce sont les rapports hommes/femmes qu'il serait intéressant d'appréhender, depuis la mise en place du voyage de noces dans les années 1840, en France, à la conception des enfants pendant les vacances : le pic des naissances en mai est à relier avec le mois d'août (Boyer, 1999), l'augmentation de la demande des divorces avec celui de septembre, lorsque la période estivale a mis en avant l'écart qui s'était creusé dans le couple, notamment quand les relations sexuelles se sont révélé insatisfaisantes (Antomarchi, 2004). Relations sexuelles et rapports au corps sont en effet deux grilles de lecture qui permettraient de cerner l'objet «tourisme et femmes». De plus, la transmission des pratiques touristiques au sein des familles devrait être approfondie comme l'ont déjà souligné les membres de l'équipe MIT (2002 : 128) détournant ainsi la très célèbre formule de Simone de Beauvoir (1949) : «On ne naît pas touriste, on le devient».

La figure de la pionnière, de l'aventurière, de la voyageuse est régulièrement mise en avant, et c'est bien souvent sous cet anglelà que la femme est visible. Pourtant, dans la problématique de «femmes et tourisme», il y a aussi le souhait de cerner, derrière l'exceptionnel, les expériences et les pratiques touristiques des femmes qui apparaissent comme les oubliées et les muettes de cette construction historique.

Une forte tradition menée par des chercheurs britanniques et américains depuis les années 1980 a produit de nombreux écrits sur les voyageuses, dont Morris (1993), Russel (1994), Robinson (2001), et Lapierre (2007). Il faut remarquer l'émergence d'une voix francophone avec Lapeyre (2007). Dans ce présent numéro de Téoros, Denis Voituret, historien, enseignant à l'Université de Guyane, met en scène une femme d'exception, Ella Maillart. Celle-ci, au-delà de la figure de l'aventurière, est aussi une professionnelle du tourisme. En devenant guide et en faisant partager son expérience à d'autres, elle apparaît comme une femme qui a joué un rôle dans la conception de produits touristiques. Anticonformiste, elle portait souvent des vêtements réservés aux hommes et fumait la pipe. Elle cherchait à profiter de la vie comme elle le voulait et défiait les conventions susceptibles d'entraver la réalisation de ses rêves. Voituret résume ainsi cette inclinaison et l'effet qu'elle eut sur les idées concernant le genre :

Par [sa] vision mixte, égalitaire du monde dans lequel elle aspire à évoluer, par son activité incessante, elle ne prête aux hommes que peu de pouvoir sur sa vie sans s'émouvoir de déclencher la peur de la confusion des rôles, le trouble dans le genre.

Si le précepte proposé par Byrne Swain et Henshall Momsen (2002 : vii) est exact, "men travel to discover the world, while women travel to discover themselves ", l'article de Voituret illustre la façon dont cela est confirmé par le type de voyageuse qu'était Maillart : «Je voulais apprendre à me connaitre. Au bout du voyage, vous vous retrouvez vous-même» (Maillart dans Voituret, ce numéro). 
Les articles sur les voyageuses tels que celui-ci, consacré à Ella Maillart, contribuent à documenter l'absence comparative de la recherche effectuée sur les voyageuses qui n'étaient pas britanniques. Il y a d'autres façons de lire ces événements historiques. Mills (1991) divise la recherche sur les femmes et les récits de voyage en deux champs : d'une part, ceux qui, sans discernement, lisent les récits de voyageuses, et d'autre part, ceux qui en font la lecture dans le cadre d'une étude critique du discours colonial. De même, Melman (2008 : 165) propose ceci :

Bon nombre de travaux sur le voyage, s'intéressant aux femmes, aux hommes, ou aux deux, ont montré que le fonctionnement du genre ne se manifeste pas uniquement dans la « différence» entre la manière dont femmes et hommes font l'expérience de l'empire et le représentent. Ces études montrent également comment le genre construit l'expérience même du voyage, la manière de percevoir la différence culturelle, de «donner un sens» et de répondre à la colonisation.

En effet, il reste beaucoup de travail à faire et de nombreuses lacunes à combler. La recherche émergente sur le tourisme, en particulier depuis les années 1990, démontre qu'elle est influencée par de nombreuses disciplines et de nombreux champs d'études. Ceux-ci ont un impact sur la façon dont nous regardons le tourisme et sur notre façon de faire des recherches sur le tourisme.

En guise de conclusion, nous voudrions faire deux observations supplémentaires, toutes deux liées au «qui» et au «comment» impliqués dans la recherche sur le genre en tourisme.

Premièrement, Margaret Byrne Swain (1995) a constaté dans les Annals of Tourism Research que la majorité des auteurs sur les questions de genre sont des femmes. Elle soulève déjà la problématique qui se présente quand nous faisons du genre une question réservée aux femmes qu'elles seules seraient amenées à comprendre et à résoudre. Aujourd'hui, malgré le petit nombre d'auteurs, il est intéressant de remarquer que la parité est presque atteinte dans ce numéro : les textes sont écrits par trois hommes et cinq femmes. Le précédent numéro de Téoros sur ce même thème était dominé par les femmes qui étaient dix à avoir contribué au numéro contre un seul homme. Le fait que les hommes se soient impliqués dans la rédaction de ce nouveau dossier de Téoros marque une évolution appréciable.

Deuxièmement, nous observons le manque criant de recherche collaborative et même comparative sur les questions de genre et tourisme. Ce manque est effectif à travers les aires géographiques, et pas seulement entre les mondes francophone et anglophone, catégorisations certes maladroites. Il l'est aussi par les champs disciplinaires. À notre connaissance, peu de recherches, si ce n'est pas de recherche, n'ont été faites pour réunir les diverses études se produisant dans ces mondes distincts de recherche sur le tourisme. Ce numéro de Téoros constitue un point de départ, par la démarche utilisée par les corédactrices invitées pour présenter, en s'intéressant à la fois aux mondes francophones et anglophones, un état de cette recherche dans différents domaines des sciences sociales.

Les discussions que nous avons eues au cours de la dernière année pour la préparation de ce numéro ont bien mis en avant l'écart qui existe autour des questions du genre et de la recherche en tourisme, du point de vue anglophone et francophone.
Suzanne de la Barre (docteure), bilingue, originaire du Québec, a une formation en géographie humaine, et son travail académique depuis ses études en maîtrise commencées en 1987 se fait exclusivement en anglais. Ses intérêts de recherche comprennent le tourisme dans les régions nordiques, les questions d'identité et de représentation et, par conséquent, l'influence du tournant critique sur les études en tourisme. Elle examine de plus en plus les interférences avec le développement économique communautaire et régional. Véronique Antomarchi (docteure) a une formation d'historienne-géographe. Son enseignement en tant que professeure agrégée dans des filières de tourisme à vocation professionnelle (brevet de technicien supérieur : Lycée René-Auffray; licence professionnelle : Université de Paris Ouest) l'a conduite à réfléchir en termes d'analyse du patrimoine et d'analyse spatiale, en mêlant histoire et géographie. Ses recherches portent notamment sur le tourisme dans les communautés inuites de l'Arctique canadien à travers la question du patrimoine et de la réappartenance.

En tant que corédactrices de ce numéro spécial, nous avons été confrontées à des défis importants. Notre objectif était d'encourager une recherche "gender-focused» et "feminist-oriented» et de susciter la réflexion et le dialogue chez les acteurs de la recherche en tourisme. Une partie de ce projet a nécessité un engagement de l'une envers l'autre pour comprendre les différents points de vue linguistique, géographique et disciplinaire que nous apportions à notre travail. Â notre manière, nous avons essayé de combler ces réalités distinctes, de trouver et de créer un terrain fertile, peut-être moins par la terminologie que nous utilisons ou par les références institutionnalisées que nous vous proposons que par notre attention commune au développement des questions du genre dans les études de tourisme.

\section{Références}

ANDRIEU, Bernard (2008) Le bronzage : une petite histoire du soleil et de la peau, Paris : CNRS éditions. $127 \mathrm{p}$.

ANTOMARCHI, Véronique (2004) «Vacances en famille : Quelques pistes de recherche sur l'évolution des pratiques et des territoires en France au cours du XXe siècle», DANS Femmes Familles Filiations, Société et Histoire, en hommage à Yvonne Knibiehler, études réunies par Marcel BERNOS et Michelle BITTON, Coll. «Le temps de l'histoire», p. 249-266. Aix-en-Provence : Presses Universitaires de Provence. ANTOMARCHI, Véronique (2007) «Peaux hâlées : renouveau des codes esthétiques et des pratiques touristiques», DANS Variations sur la peau, sous la direction de Stéphane HEAS et Laurent MISERY, Coll. «Le corps en question », p. 99-109. Paris : L'Harmattan.

ATELJEVIK, Irena; Annette PRITCHARD et Nigel MORGAN (éd.) (2002) The Critical Turn in Tourism Studies: Innovative Research Methodologies, Boston : Elsevier. 428 p.

BARD, Christine (dir.) (2004) Le genre des territoires : masculin, féminin, neutre, Angers : Presses universitaires d'Angers. $348 \mathrm{p}$.

BARTHE Francine et Claire HANCOCK (2006) Géographie et cultures, n 54 , «Le genre : constructions spatiales et culturelles», Paris, 146 pages.

BERTHO-LAVENIR, Catherine (1996) La roue et le stylo, comment nous sommes devenus touristes, Paris: Odile Jacob. 438 p.

BLIDON, Marianne (2008) «À travers l'espace de la méthode : les dimensions du terrain en géographie», Arras, actes mis en ligne sur le site HAL-SHS <http://halshs.archives-ouvertes.fr/halshs-00422392/en/>. 
BLIDON, Marianne et Sébastien ROUX (2009) «Appel à communication», DANS L'espace politique, parution 2011.

BOYER, Marc (1999) «Le tourisme, une épistémologie spécifique», suivi de «L'historien et le tourisme», Les Cahiers du G.D.R, n 2, p. 7-25.

BYRNE SWAIN, Margaret et Janet HENSHALL MOMSEN (éd.) (2002) Gender/Tourism/Fun?, département d'anthropologie, Davis : Université de Californie. $250 \mathrm{p}$.

BYRNE SWAIN, Margaret (1995) «Gender in Tourism», Annals of Tourism Research, vol. 22, n 2 , p. 247-266.

CAUVIN-VERNER, Corinne (2009) «Du tourisme culturel au tourisme sexuel : les logiques du désir d'enchantement», Cahiers d'études africaines, «Tourismes La quête de soi par la pratique des autres», $n^{\circ}$ 193-194, Paris : EHESS, p. 123-146.

CAZES, Georges (1992) Fondements pour une géographie des tourismes et des loisirs, Paris : Bréal. 189 p.

CHABAUD-RYCHTER, Danielle; Virginie DESCOUTURES; Anne-Marie DEVREUX et Eleni VARIKAS (2010) Sous les sciences sociales, le genre. Relectures critiques, Paris : La Découverte. 512 p.

CHEVREL, Claudine; Béatrice CORNET et André RAUCH (2006) Les vacances, un siècle d'images, des milliers de rêves 1860-1960, Paris : Bibliothèques. $208 \mathrm{p}$.

CORBIN, Alain (dir.) (1995) L'avènement des loisirs 1850-1960, Paris : Aubier. $472 \mathrm{p}$.

COUSIN, Saskia et Bertrand REAU (2009) Sociologie du tourisme, Coll. «La Découverte», Paris : Repères, 128 p.

DE BEAUVOIR, Simone (1949) Le deuxième sexe, t. 1, Paris. Gallimard. 401 p. DENZIN, Norman et Yvonne LINCOLN (1994) «Introduction: Entering the field of qualitative Research", DANS Handbook of qualitative research, second edition, sous la direction de N. Denzin et Y. Lincoln, p. 1-17. Londres : Sage Publications.

DENZIN, Norman et Yvonne LINCOLN (2000) «Introduction: The Discipline and practice of qualitative research", DANS Handbook of qualitative research, second edition, sous la direction de N. Denzin et Y. Lincoln, p. 1-28. Londres: Sage Publications.

ÉQUIPE MIT (2001) Tourismes 1, Lieux communs, Paris : Mappemonde, Belin, $320 \mathrm{p}$.

GAGNON, Sylvie (1997) «Des réalités roses et moins roses... incontournables», Téoros, vol. 16, n 3, «Tourisme et femmes», Québec: UQAM.

HANCOCK, Claire (2004) «L'idéologie du territoire, une discipline masculiniste», DANS Le genre des territoires, masculin, féminin, neutre, sous la direction de Christine BARD, p. 165-174. Angers : Presses Universitaires d'Angers.

JAURAND, Emmanuel et Hubert DE LUZE (2004) «Ces plages où les genres s'affichent ? Les territoires du nu sur la Côte d'Azur ", DANS Le genre des territoires: masculin, féminin, neutre, sous la direction de Christine BARD, p. 227-240. Angers : Presses de l'Université d'Angers.

KAUFMANN, Jean-Claude (1995) Corps de femmes, regards d'hommes, Sociologie des seins nus, Paris : Nathan. 240 p.

KINNAIRD, Vivian et Derek HALL (éd.) (1994) Tourism: A Gender Analysis, Grande-Bretagne : John Wiley \& Sons. 236 p.

LABOURIE-RACAPÉ, Annie (2004) «Genre et territoire, quelles questions», Espaces populations sociétés, vol. 1, Lille, p. 113-119.

LAPIERRE, Alexandra (2007) Women Travelers: A Century of Trailblazing Adventures 1850-1950, Paris : Flammarion. 240 p.

LAPEYRE, Françoise (2007) Le roman des voyageuses françaises 1800-1900, Paris : Payot. 270 p.

LETHERBY, Gale et Gillian REYNOLDS (2009) Gendered Journeys, Mobile Emotions, Londres : Ashgate Publishing. 236 p.
MELMAN, Billie (2008) «Actualité de la recherche, Orientations historiographiques : Voyage, genre et colonisation", Clio, Histoire, Femmes et Sociétés, $\mathrm{n}^{\circ}$ 28, «Voyageuses», Toulouse : Presses Universitaires du Mirail, p. $159-185$.

MILLS, Sara (1991) Discourses of Difference: An Analysis of Women's Travel Writing and Colonialism, Londres : Routledge. $232 \mathrm{p}$.

MORGAN, Nigel et Annette PRITCHARD (1998) Tourism Promotion and Power: Creating Images, Creating identities, Chichester : Wiley. 272 p.

MORRIS, Mary (1993) Maiden Voyages: Writings of Women Travelers, New York : Vintage Books. $464 \mathrm{p}$.

ONU (2009) «L'égalité entre hommes et femmes au cœur du travail décent : conférence internationale du Travail, 98 ession ", Rapport VI, 17 p.

ORY, Pascal (2008) L'invention du bronzage, Paris : éditions Complexes. $132 \mathrm{p}$.

PIVETEAU, Jean-Luc (1996) «Notre territorialité n'est-elle pas essentiellement masculine?», Géographie et cultures, n 20, Paris, p. 69-80.

PHILLIMORE, Jenny et Lisa GOODSON (2004) «Progress in qualitative research in tourism», DANS Quaitative Research in Tourism: Ontologies, Epistemologies and Methodologies sous la direction de J. Phillimore et Lisa Goodson, p. 3-30. Londres et New York : Routledge.

PRITCHARD, Annette et Nigel MORGAN (2000) «Privileging the Male Gaze: Gendered Tourism Landscapes", Annals of Tourism Research, vol. $27, n^{\circ} 4$, p. 884-905.

PRITCHARD, Annette; Morgan NIGEL; Irena ATELJEVIC et Candice HARRIS (2007) Tourism and Gender: Embodiment, Sensuality and Experience, Londres : CAB International. $352 \mathrm{p}$.

PRITCHARD, Annette (2005) «Gender and Sexuality in Tourism Research», DANS A Companion to Tourism, sous la direction de A. Lew, C.M. Hall et A.M. Williams, p. 316-326. Oxford, UK : Blackwell Publishing.

RAO, N. (1995) «Commoditisation and Commercialisation of Women in Tourism: Symbols of Victimhood», Contours, vol. 7, nº 1, p. 30.

RAUCH, André (1996) Vacances en France de 1830 à nos jours, Paris : Hachette. 280 p.

RIOT-SARCEY, Michèle (dir.) (2010) De la différence des sexes : le genre en histoire, Paris : Larousse. 287 p.

ROBINSON, Jane (2001) Unsuitable for Ladies: An Anthology of Women Travellers, Oxford : Oxford University Press. 496 p.

ROGERS, Rebecca et Françoise THÉBAUD (2008) Clio, Histoire, Femmes et Sociétés, $n^{\circ} 28$, «Voyageuses», Toulouse : Presses Universitaires du Mirail, $319 \mathrm{p}$.

RUSSEL, Mary (1994) The Blessings of a Good Thick Skirt: Women Travellers and their World, Londres : Harper Collins. 240 p.

SAMUEL, Nicole (1997) «Tourisme et femmes», Téoros, vol. 16, nº 3, «Les femmes en situation de vacances».

STASZAK, Jean-François et Béatrice COLLIGNON (2004) «Que faire de la géographie postmoderniste?», L'Espace Géographique, vol. 1, t. 33, Paris, p. 38-41.

STOCK, Mathis et Anne VOLVEY (2003) «Sexualité», DANS Dictionnaire de la géographie et de l'espace des sociétés, sous la direction de Jacques LEVY et Michel LUSSAULT, Paris : Belin, 1032 p.

STOCK, Mathis (coord.) (2003) Le tourisme, acteurs, lieux, enjeux, Paris : Belin. 303 p.

TÉOROS (2008) Dossier : Une science du tourisme?, vol. 27, nº 1, printemps, Québec: UQAM.

URBAIN, Jean-Didier (1991) L'idiot du voyage, Histoires de touristes, Paris : Payot. 353 p.

WITIA (2010) «Women in Tourism International Alliance Ltd», <http:// witia.org/>, consulté le 15 septembre 2010. 